\title{
Number of Live Female Fetuses
}

National Cancer Institute

\section{Source}

National Cancer Institute. Number of Live Female Fetuses. NCI Thesaurus. Code C124622.

A measurement of the number of live female fetuses present in the uterus. 